\title{
Heart Rate Detection System Based on Android OS
}

\author{
Dejun $\mathrm{Li}^{\mathrm{a}}$, Zheng Xie ${ }^{\mathrm{b}}$, Xiaobo $\mathrm{Lu}^{\mathrm{c}}$ \\ School of Wuhan Textile University, Wuhan 430073, China \\ adj@wtu.edu.cn, b510819030@qq.com, ${ }^{\text {c}} 1151999045 @ q q . c o m$
}

Keywords: wearable, MCU, bluetooth, heart rate detection.

\begin{abstract}
This design is wearable digital heart rate meter, the acquisition and transformation use of heart rate data in lower computer is STC12C5A60S2 MCU with high speed 51 kernel, the heart rate sensor is used in photo-electric reflective analog sensor, which adopted method combine hardware and software filtering and greatly improves the accuracy of heart rate signal detection of the human body. And the final data is passed to the android mobile phone through bluetooth, human body heart rate of real-time monitoring function is realized in android mobile phone.
\end{abstract}

\section{Introduction}

In recent years, our country enter into aging society gradually, with the growth of the age, the probability of cardiovascular disease sickness will be greatly increased, the body's heart rate signals is very important in biomedical signal, the heart rate signal processing is a research hotspot rapidly develop both at home and abroad in recent years. With the development of signal processing technology and computer processing technology, people hope to apply modern technology into the research of heart rate signal so that more scientific and more objectively reveals the essence and characteristics of heart rate. Since the Android OS is launched, it gradually enlarges the market share with obvious advantages, its high call and like the sun in broad daylight, which is in the booming development stage. The heart rate detection system is using android phones as heart rate display terminal, and method combined hardware and software filtering is adopted, it can accurately obtain the heart rate value, and can data is displayed in the android mobile phone, it is a mobile portable wearable device.

\section{The design heart rate detection system}

The pulse signal likes other biological electrical signals, which has weak signal, low frequency and easily be interfered and so on, the measurement method of pulse signal has 3 kinds generally: the first is extracted from the ECG signal, the second is the wave detected from the pressure sensors of measuring blood pressure calculate the pulse frequency, the third is photoelectric volumetric method. Since former two methods is long time measured, which will bring the physical discomfort to tester, so photoelectric volumetric method is used in this time.

PhotoPlethysmoGraphy (PPG) is a kind of noninvasive detection methods by photoelectricity means checking blood volume change in living tissue, when beam with certain wavelengths irradiating fingers skin surface, the beam will be transmitted to the photoelectric receivers through the way of transmission or reflection, in this process, because of fingers skin muscles and blood absorption attenuation function, the light intensity detected by detector will weaken, the skin tissue muscles keep constant in the absorption of light in the whole blood circulation.In this system on empty baud rate is 115200 , data from the digits for eight, bluetooth matching password for 123456. When bluetooth mobile phone search to XM - $10 \mathrm{~b}$, password after successful matches can establish a connection, and then you can with STC12C5A60S2 serial communication.

System processor adopts STC12C5A60S2 MCU, it uses 51 kernel, compared with the traditional $89 \mathrm{MCU}$, it has unique $12 \mathrm{~T}$ high speed operation mode, and the internal chip is abound in peripherals, it has 8 input 10 decimal AD conversion module, it is not necessary to connect externally AD conversion chip to turn the detected voltage signal into digital signal and then transmit to MCU. Here is the heart rate sensor PulseSensor, The output voltage of PulseSensor is between $3 \mathrm{~V}$ to $3.3 \mathrm{~V}$; just in 
the range of MCU receives analog, no longer need to plus a signal disposal circuit. After turn analog voltage signals transmitted by PulseSensor into digital signals, after get the data will be the heart rate after numerical display LCD screen in 1602, at the same time, the data through a serial port transmit to serial port turn bluetooth module HC- 06, mobile phone open bluetooth function, and after successfully connect XM-10B, it can receive the heart rate data and displayed on the phone. Overall framework diagram of system are shown in Fig.1 below:

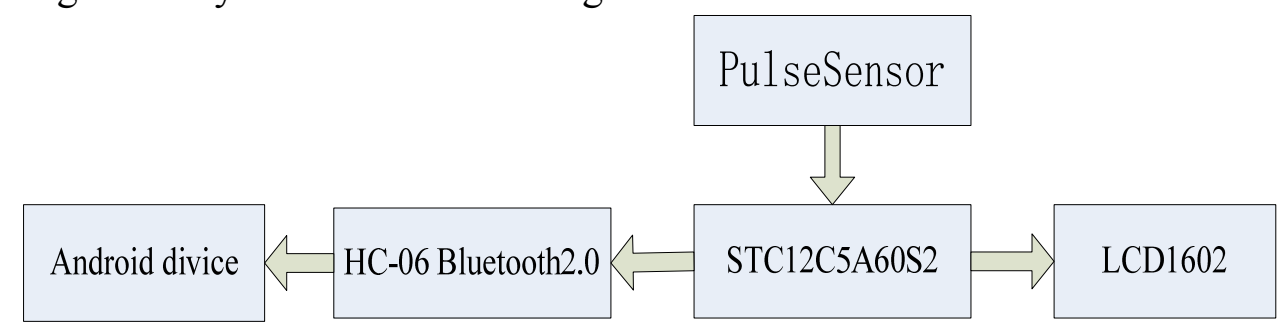

Fig.1 Overall framework diagram of system

\section{The hardware design of heart rate detection system}

\subsection{PulseSensor sensors}

PulseSensor is a photoelectric diffuse type analog sensor to measure pulse heart rate. The Fig. 2 is schematic diagram of PulseSensor sensor module, the voltage of sensor module amplifies 330 times, ECG is generally at the millivolt level, after 330 times amplification, the voltage signal can be well detected by MCU. Power supply voltage is between 3.3 and $5 \mathrm{~V}$, because the MCU used a 5 v power supply, for the sake of convenience of power circuit design here, the sensor module adopts $5 \mathrm{~V}$ power supply way.

The heart rate detection device is wore on the finger, the earlobe and so on, analog signals were collected through a wire connection can be passed to the STC12C5A60S2 MCU to be converted into digital signals. After STC12C5A60S2 MCU simply calculates, the heart rate value can be obtained.

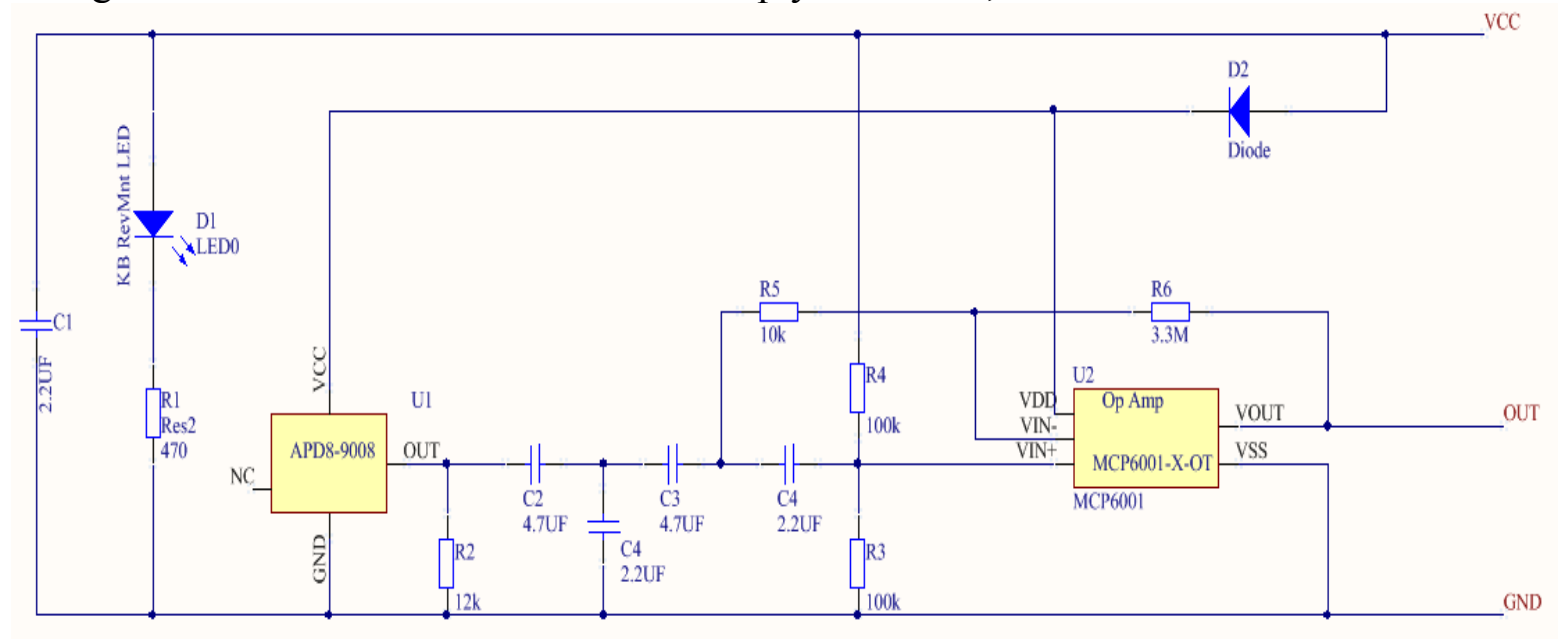

\subsection{MCU smallest system}

Fig. 2 The principle diagram of PulseSensor sensor module

The minimum system circuit of STC12C5A60S2 includes power supply circuit, clocking circuit and reset circuit, it is necessary condition for MCU to work, STC12C5A60S2 is a high speed 51 kernel MCU, except have high working frequency, compared with the traditional 51MCU, it has plenty of chip resources, its internal contains 8 input 10 decimal AD conversion module, so there is no need to connect $\mathrm{AD}$ chip.

\subsection{Bluetooth wireless communication module}

MCU and android mobile phone communications in this system adopt widely used in market XM-10B bluetooth serial communication module, the module can turn MCU serial data into the bluetooth data and transmit to the android mobile phone, XM-10B bluetooth serial module adopts bluetooth 4.0BLE protocol, compared with general bluetooth 2.0 communication, bluetooth 4.0 has 
very low power consumption. XM- 10B and STC12C5A60S2 is connected through serial, the serial communication parameters such as baud rate, transmission digits, parity check, bluetooth matching password and so on, it is set by the AT command.

\section{The software design of heart rate test system}

\subsection{MCU software design}

When the pulse beating is completed, the pulse will also have a mild beating, the detected wave of beating is not necessary, we call it a heavy wave, it need to be filtered and removed.The ware goes like Fig.3. In STC12C5A60S2 side algorithm, we will regard pulse wave less than 0.6 heart beating as heavy wave. STC12C5A60S2 calculates time difference between every two pulse peak value to get the heart time, here variable beatTime represent time between heartbeat, then beatTime is divided by 60 , the number of heart beats every minute can be obtained. After calculating the number of heart, then every $10 \mathrm{~ms}$ data is transmitted from serial port to XM-10B bluetooth serial communication module, and then the android mobile terminal can receive data. The data take beat as the frame head beat + heart number to transmit out

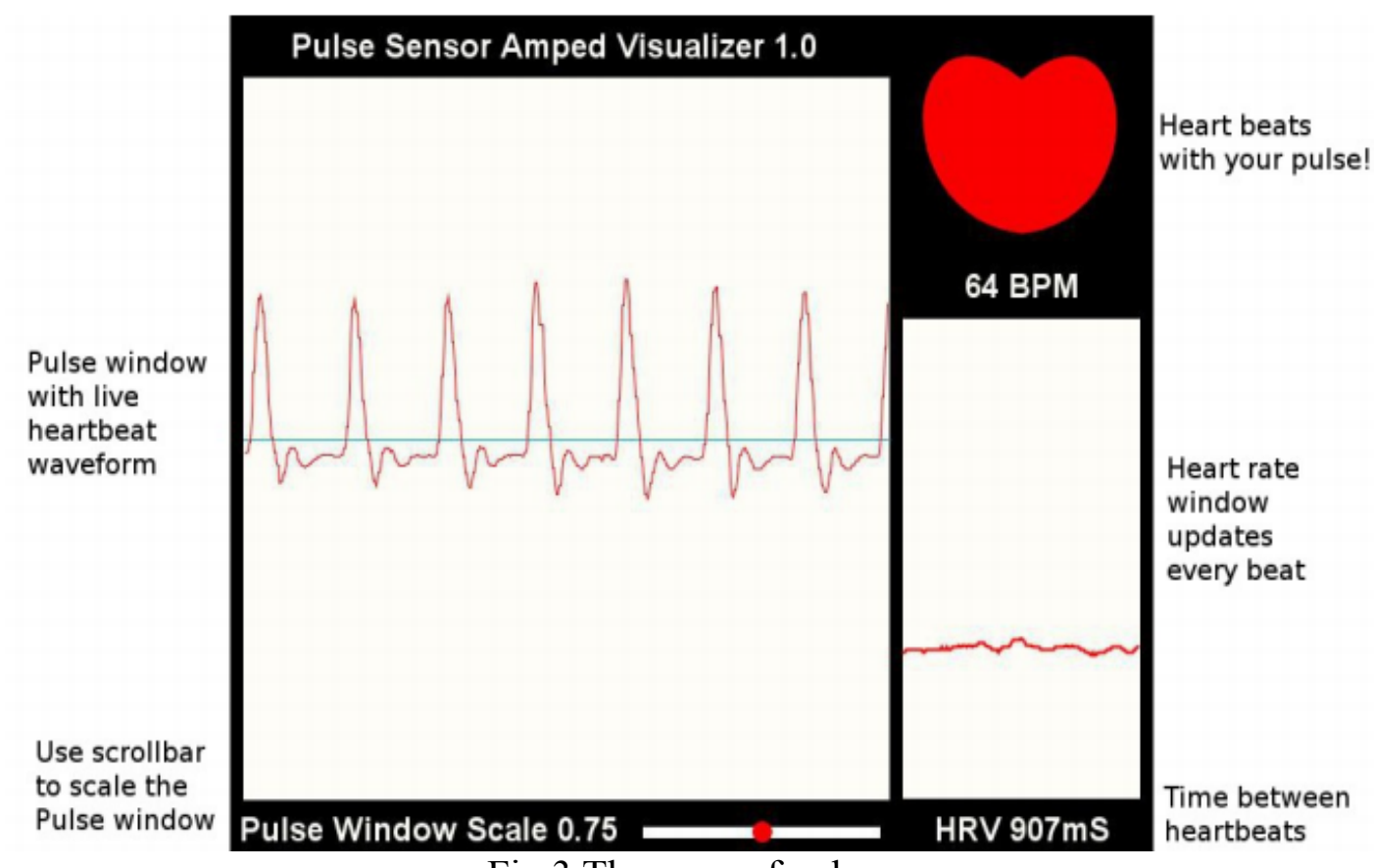

Fig.3 The ware of pulse

\subsection{The software design of Android terminal}

Single chip microcomputer system sends data after XM - $10 \mathrm{~b}$ a serial port turn bluetooth module will send data to the android mobile phone, then STC12C5A60S2 with AnZhuoDuan established communications android phones .The development of applications of Android is used java.The UI of android is used xml. Because Android terminal receive MCU data is by bluetooth, so the bluetooth permissions in the android terminal must be open, namely in the AndroidManifest. XML document add the android. permission, bluetooth and android permission. Bluetooth_Admin Permission. In the main interface, bluetooth data thread is opened. The received data are carried out process in the thread, if beat in the beginning, then take the data behind beat, then open a message, the corresponding control will display heartbeat value.

\section{Conclusion}

Along with our country entering an aging society, as well as rapid popularization of mobile phone and other intelligent terminals, more and more portable health software and mobile phone are into 
people's pockets, more and more people attach great importance to this portable software related to human health, this will be a very good development.

\section{References}

[1] Han Xingguo, Su Qingyong, Wang Weiqing, Based on the STC89C52 single-chip microcomputer intelligent fan control system design, Equipment Manufacturing Technology, 2013, 01.

[2] Jie Zhang, Jianjun Sun, Portable heart rate tester is designed based on single-chip microcomputer, Electronic World, 2012, 06.

[3] Yan Han, Digital heart rate tester is developed, NeiJiang Technology, 2004, 01.

[4] Xiaokun Feng, Guang Yang, Xiaofeng Wang, The wearable computer development present situation and existing problems of research, 2011, 29. 